\title{
Efecto de las deficiencias y excesos de fósforo, potasio y boro en la fisiología y el crecimiento de plantas de aguacate (Persea americana, cv. Hass)
}

\author{
Effect of deficiencies and excesses of phosphorus, \\ potassium and boron on the physiology and growth of \\ avocado (Persea americana, cv. Hass) plants
}


\section{RESUMEN}

Para conocer el efecto de los nutrientes minerales potasio $(\mathrm{K})$, boro $(\mathrm{B})$ y fósforo $(\mathrm{P})$, sobre variables fisiológicas y de crecimiento en aguacate var. Hass, se evaluaron siete tratamientos y cuatro réplicas con tres niveles de dosis: (1) deficiencia del respectivo elemento: $50 \%$ de la fertilización completa, (2) completa o tratamiento control: $100 \%$ y (3) exceso con un 150\% por encima de la fertilización completa, dispuestos en un diseño completamente aleatorio. Las plantas crecieron en bolsas de polietileno, con suelo, y bajo condiciones de invernadero. Se realizó fertilización y riego semanal. Las variables evaluadas fueron el área foliar (AF), número de hojas (NH), contenido relativo de clorofilas (CC), eficiencia máxima fotoquímica del fotosistema II (Fv/ Fm) y transpiración (E), además se describió la sintomatología de deficiencias o excesos en hojas. Se encontró que los valores de AF, NH y CC fueron menores en relación con el control. De igual forma, Fv/Fm y la E fueron menores con respecto al control, sugiriendo probable estrés ocasionado por los tratamientos; presentándose en mayor proporción para P, seguido de K y B. Los signos visuales de estrés nutricional se observaron de forma más clara en las plantas sometidas a deficiencia que en aquellas sometidas a exceso, siendo acorde a lo reportado para esta variedad.



Palabras clave adicionales: nutrición mineral, área foliar, fluorescencia de la clorofila, transpiración, síntomas de deficiencia y exceso, frutales.

Facultad de Ciencias, Departamento de Biología, Laboratorio de Fisiología y Bioquímica Vegetal, Universidad Nacional de Colombia, Bogotá (Colombia). ORCID Novoa, M.A.: 0000-0001-5817-4714; ORCID Melgarejo, L.M.: 0000-0003-3148-1911

Facultad de Ciencias Agrarias, Departamento de Agronomía, Universidad Nacional de Colombia, Bogotá (Colombia). ORCID Miranda, D.: 0000-0001-9861-6935

Autor para correspondencia.1mmelgarejom@unal.edu.co 


\section{ABSTRACT}

In order to observe the effects of nutrients: potassium (K), boron (B) and phosphorus (P), on physiological and growth variables in avocado var. Hass, seven treatments and four repetitions with three dose levels were evaluated: (1) deficiency of the respective element: $50 \%$ or half of the complete fertilization, (2) complete or control treatment: $100 \%$, and (3) excess of the respective element, $150 \%$ above the complete fertilization, arranged in a completely random design. The plants were grown in polyethylene bags with soil and cultivated under greenhouse conditions; weekly fertilization and irrigation were also carried out. The evaluated variables included leaf area (LA), number of leaves (NL), relative chlorophyll content (CC), maximum photochemical efficiency of PSII (Fv/Fm) and transpiration (E); the symptomatology of deficiencies and excesses were described in the leaves. The LA, NL and CC values were lower in relation to the control. Similarly, the Fv/Fm and E were lower compared to the control, suggesting probable stress caused by the treatments, presenting a greater proportion of $\mathrm{P}$, followed by $\mathrm{K}$ and $\mathrm{B}$. The visual signs of nutrient stress were observed more clearly in the plants subjected to deficiency than in those subjected to excess, consistent with what has been reported for this variety

Additional key words: mineral nutrition, leaf area, chlorophyll fluorescence, transpiration, deficiency and excess symptoms, fruits.

Fecha de recepción: 06-04-2018 Aprobado para publicación: 30-05-2018

INTRODUCCIÓN

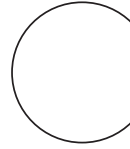

El aguacate (Persea americana Mill.) es el cuarto fruto tropical más importante para el consumo humano a nivel mundial, estimándose una producción global de 5,6 millones de toneladas anuales (FAO, 2016). Una de las variedades de Persea americana que se destaca por su actual demanda mundial es la variedad Hass (Bartoli, 2008). Esta presenta óptima calidad en sus frutos, alto rendimiento, maduración tardía (Bernal y Díaz, 2005) y se adapta a condiciones subtropicales y tropicales, con temperaturas de 5 a $19^{\circ} \mathrm{C}$ y altitudes entre los 1.600 y 2.100 msnm (Mejía, 2010; ICA, 2012).

Los mayores cultivos de aguacate se encuentran en América, donde se destaca México como el primer productor mundial. Colombia ocupa el quinto lugar de los países con mayor área cultivada de aguacate en el mundo (FAO, 2016). Sin embargo, llama la atención que, a pesar del gran potencial que tiene el aguacate como producto exportable, el país no haya podido consolidarse como un exportador neto (Yabrudy, 2008). Además, que internacionalmente Colombia no figure como un productor importante (Agronet, 2016).

Como limitantes en la producción, incide el bajo desarrollo tecnológico del cultivo, los deficientes canales de comercialización y las pérdidas causadas por plagas (ICA, 2012). Así como la alta demanda interna y la falta de prácticas agrícolas apropiadas que dificultan el acceso a los mercados internacionales (Ríos y Tafur, 2003).

A su vez, Maldonado et al. (2013) afirman que algunos de los principales limitantes de la producción son las diferencias entre el rendimiento promedio y los máximos registrados, los cuales estarían indicando la existencia de factores restrictivos de la producción que no han sido identificados apropiadamente y que requieren ser investigados para proponer mejores alternativas, que conduzcan a la obtención de altos rendimientos y óptima calidad de fruto, como es el caso de la nutrición mineral.

De acuerdo con Salazar et al. (2009) las recomendaciones de fertilización implementadas en los sistemas de producción de aguacate son generales y suelen ser usadas sin considerar las variaciones en la fertilidad de los suelos, ni la condición nutricional de los árboles, o el efecto de esta sobre los mismos. Esto genera como consecuencia que se apliquen menos nutrientes de los necesarios para maximizar la producción o que se incurra en una aplicación excesiva, lo que conduce a la aparición de desbalances nutricionales, que 
además de limitar la productividad pueden producir contaminación de los recursos naturales (Salazar y Lazcano, 2003).

De esta manera es posible afirmar, que la adecuada nutrición mineral de un cultivo está determinada, entre otros, por el conocimiento de los requerimientos de la planta y por la cantidad y disponibilidad de nutrimentos del suelo (Salas, 2002).

Es así, como resulta de gran importancia conocer el efecto de la aplicación excesiva o deficitaria de nutrientes, como fósforo, potasio y boro, sobre variables de crecimiento y desarrollo, así como su aspecto sintomatológico en plantas de aguacate, cv. Hass, en su fase vegetativa.

\section{MATERIALES Y MÉTODOS}

\section{Localización y material vegetal}

El estudio se llevó a cabo en los invernaderos de la Universidad Nacional de Colombia, Bogotá (4³8'09,1" N y $74^{\circ} 05^{\prime} 13,6^{\prime \prime}$ W) a $2.640 \mathrm{msnm}$, temperatura del aire entre $18-20^{\circ} \mathrm{C}$ y humedad relativa entre $60-90 \%$. El material vegetal consistió en plantas de aguacate (Persea americana, cv. Hass, injertado sobre patrón Criollo) de 8 meses de edad, las cuales se sembraron en bolsas de polietileno $(0,5 \times 0,7 \mathrm{~m})$ con suelo proveniente del municipio El Rosal-Cundinamarca (Tab. 1).
Las plantas se mantuvieron en condiciones óptimas de irrigación. La fase de laboratorio se desarrolló en el Laboratorio de Fisiología Vegetal del Departamento de Biología de la Facultad de Ciencias de Universidad Nacional de Colombia, Bogotá.

\section{Diseño experimental}

Se empleó un diseño completamente aleatorizado (DCA). Se establecieron siete tratamientos y cuatro réplicas de cada uno: tres niveles o dosis de los nutrientes potasio $(\mathrm{K})$, fósforo $(\mathrm{P})$ y boro (B): 50,100 , $150 \%$ (Tab. 2). Se realizaron aplicaciones de solución nutritiva de manera semanal. Las fuentes utilizadas fueron: ácido fosfórico líquido, fosfato monopotásico y ácido bórico. En total se evaluaron 84 plantas en 13 muestreos con una frecuencia de $15 \mathrm{~d}$ (durante 6,5 meses). En cada muestreo se evaluaron cuatro plantas de cada tratamiento.

\section{Análisis estadístico}

La organización de los datos y el análisis gráfico se realizó con el programa Excel 2016 (Microsoft Office, 2016) y SigmaPlot 10.0. El análisis de los datos se ejecutó con el programa SAS 9.1 (Statistical Analysis System), utilizando la prueba de comparación de Duncan con una significancia del $95 \%$. Los datos que no cumplieron supuestos de normalidad y homogeneidad de varianza fueron sometidos a la transformación de Box y Cox.

\section{Tabla 1. Características fisicoquímicas del suelo empleado en el estudio.}

\begin{tabular}{|c|c|c|c|c|c|c|c|c|c|c|}
\hline \multirow{2}{*}{$\mathrm{pH}$} & \multirow{2}{*}{ CE } & $\mathrm{CO}$ & $\mathrm{N}$ & $\mathrm{Ca}$ & K & $\mathrm{Mg}$ & $\mathrm{Na}$ & $\mathrm{Al}$ & CICE & $\mathrm{CIC}$ \\
\hline & & \multicolumn{2}{|c|}{$\%$} & \multicolumn{7}{|c|}{$\mathrm{cmol}_{(+)} \mathrm{kg}^{-1}$} \\
\hline 5,5 & - & 7,92 & 0,68 & 8,43 & 0,56 & 2,57 & 0,54 & 0,22 & 12,3 & - \\
\hline$P$ & $S$ & $\mathrm{Cu}$ & $\mathrm{Fe}$ & $\mathrm{Mn}$ & $\mathrm{Zn}$ & $B$ & $\mathrm{Ar}$ & $L$ & $\mathrm{~A}$ & vtur \\
\hline \multicolumn{7}{|c|}{$\mathrm{mg} \mathrm{kg}^{-1}$} & \multicolumn{3}{|c|}{$\%$} & xtura \\
\hline 12,2 & 60,5 & 0,32 & 15,7 & 4,87 & 2,24 & $<0,12$ & 10 & 32 & 58 & FA \\
\hline
\end{tabular}

Tabla 2. Fertilización de las plantas de aguacate de acuerdo con los tratamientos.

\begin{tabular}{|c|c|c|c|c|c|c|c|}
\hline \multirow{2}{*}{ Nutriente } & Control & P150 & P50 & K150 & K50 & B150 & B50 \\
\cline { 2 - 8 } & \multicolumn{7}{|c|}{ g/planta-semana } \\
\hline $\mathrm{P}_{2} \mathrm{O}_{5}$ & 8,6 & 12,9 & 4,3 & 8,6 & 8,6 & 8,6 & 8,6 \\
\hline $\mathrm{K}_{2} \mathrm{O}$ & 11,5 & 11,5 & 11,5 & 17,3 & 5,8 & 11,5 & 11,5 \\
\hline $\mathrm{B}$ & 1,0 & 1,0 & 1,0 & 1,0 & 1,0 & 1,5 & 0,5 \\
\hline
\end{tabular}




\section{Análisis de crecimiento}

Se realizaron mediciones directas del número total de hojas de cuatro plantas por tratamiento y el área foliar de tres plantas por tratamiento, esto debido al número total de unidades experimentales disponibles para realizar muestreos destructivos. El área foliar se determinó mediante el software Image ${ }^{\circledR} 1-45 \mathrm{~s}$ (http://imagej.nih.gov/ij/).

\section{Cuantificación relativa de clorofilas}

Se determinó realizando cuatro mediciones en tres hojas completamente expandidas de cuatro plantas diferentes, de cada tratamiento, con un medidor de clorofila portátil (SPAD 502DL; Konica Minolta, Tokio, Japón).

\section{Fluorescencia de la clorofila}

Se realizaron mediciones de la eficiencia máxima fotoquímica del PSII (Fv/Fm) empleando el equipo fluorómetro no modulado modelo Handy PEA (Hansatech Instruments, Pentney, UK) en tres hojas del tercio medio del dosel de cuatro plantas diferentes, por tratamiento. Las hojas fueros adaptadas a oscuridad por 30 min para poder realizar la medición en las primeras horas de la mañana (6:00 a 11:00 h).

\section{Intercambio de gases}

Se evaluó en las primeras horas de la mañana (6:00 a 11:00 h), el intercambio de gases en tres hojas del tercio medio del dosel de cuatro plantas diferentes, monitoreando variables como temperatura de la hoja, resistencia estomática, transpiración y radicación fotosintéticamente activa, empleando el equipo porómetro (LI-1600; Li-Cor, Lincoln, NE).

\section{Sintomatología}

Se realizó la descripción de la sintomatología de las deficiencias y excesos de nutrientes, tanto en hojas jóvenes como adultas.

\section{RESULTADOS Y DISCUSIÓN}

\section{Análisis de crecimiento}

\section{Número de hojas (NH)}

Potasio. Se presentaron diferencias significativas $(P \leq 0,05)$ a los 180 días después del trasplante (ddt) entre K50 y el tratamiento K100, y entre todos los tratamientos a los $195 \mathrm{ddt}$. A lo largo del ciclo, K50 presentó el más bajo número de hojas (62,4 hojas), siendo $14 \%$ menor con respecto a K150 (72,9 hojas) y $18 \%$ con K100 (76,1 hojas) (Fig. 1A). De acuerdo con Jordan y Pellerin (2004) el menor número de hojas por deficiencia de $\mathrm{K}$, puede estar relacionado con el retraso en la aparición de las mismas, así como una ligera aceleración en el proceso de senescencia.

Boro. Se evidenciaron diferencias estadísticas $(P \leq 0,05)$ entre B50 y B150 a los $90 \mathrm{ddt}$ y entre todos los tratamientos a los 195 ddt (Fig. 1B). En general, a lo largo del ciclo de evaluación el tratamiento B50 (82,3 hojas) presentó el mayor $\mathrm{NH}$, seguido de B100 (76,1 hojas) y B150 (68,9 hojas). La superioridad de B50 en esta variable fue del $8 \%$ más sobre B100 y 19\% con respecto a B150. El menor número de hojas puede asociarse con el efecto defoliante del exceso de este nutriente (Retamales, 2005). El mayor NH en las plantas sometidas a B50, posiblemente se relaciona con la existencia de entrenudos muy cortos, lo cual generó mayor número de nudos (B50: 106 nudos; B100: 97 nudos y B150: 94 nudos) y hojas por unidad de área. Estos resultados coinciden con lo observado por Sindoni et al. (1994) en ajonjolí (Sesamum indicum), donde la deficiencia severa de B activó las yemas axilares acortando los entrenudos apicales, dando a la arquitectura de la planta y a la disposición de las hojas un aspecto de roseta.

Fósforo. Se presentaron diferencias significativas $(P \leq 0,05)$ entre tratamientos desde los $150 \mathrm{ddt}$ hasta el final del experimento (Fig. 1C). P50 presentó los valores más bajos para esta variable (62,8 hojas), seguido de P100 (76,1 hojas) y P150 ( 84,9 hojas). El $\mathrm{NH}$ de P50 fue 18\% menor en relación al de P100 y $26 \%$ con respecto al de P150. De acuerdo con el IPNI (2010), el efecto más acentuado de la deficiencia de fósforo en las plantas es la reducción en el número de hojas. Según Kass (1998), el fósforo al ser un nutriente que se encuentra en fuertes concentraciones en los tejidos meristemáticos, ser fuente primaria de energía vía ATP, formar parte de las coenzimas NAD y NADP y participar activamente en la síntesis de proteínas, si hiciese falta, se produciría menor crecimiento y emisión de nuevos órganos vegetativos. Por otra parte, Oliva et al. (1995) afirman que las concentraciones superiores de $\mathrm{P}$, no generan un aumento significativo en el $\mathrm{NH}$.

\section{Área foliar (AF)}

El comportamiento del AF mostró una tendencia ascendente en todos los puntos de muestreo. La máxima 




Figura 1. Deficiencias y excesos nutricionales sobre el número de hojas de aguacate 'Hass'. A. Potasio (K); B. Boro (B) y C. Fósforo (P). Promedios con letras distintas indican diferencia significativa entre tratamientos, de acuerdo a la prueba de Duncan $(P \leq 0,05)$.
AF de las plantas durante el ciclo de cultivo se presentó en aquellas con el tratamiento P150 $\left(9.943,6 \mathrm{~cm}^{2}\right)$, seguido del control $\left(9.346,8 \mathrm{~cm}^{2}\right)$ a los $180 \mathrm{ddt}$. Para este mismo periodo de tiempo, el AF mínimo evidenciado en las plantas del tratamiento $\mathrm{K} 50\left(5.343,3 \mathrm{~cm}^{2}\right)$ mostró una disminución del $43 \%$ comparado con el control (Fig. 2A).

El potasio es el nutriente con mayor efecto sobre la velocidad de crecimiento, principalmente del área foliar (Fairhurst, 1999). En tanto, Pillimue et al. (1998) afirmaron que la disminución en el AF presentada por la deficiencia de K, puede ser debida al importante papel que este nutriente cumple para equilibrar la absorción de nitrógeno, mantener la turgencia de las hojas y facilitar el transporte de asimilados.

Por otro lado, las plantas de los tratamientos B150 $\left(7791,8 \mathrm{~cm}^{2}\right)$ y $\mathrm{P} 50\left(8671,1 \mathrm{~cm}^{2}\right)$ presentaron una reducción del AF del 17 y 7\%, respectivamente, frente al control (Fig. 2B y 2C). Para el caso del fósforo, su reducción puede ser indicio del importante papel que desempeña este elemento en los procesos energéticos, de tal manera que su deficiencia haya disminuido la formación de hojas. Por su parte, la reducción del AF en el tratamiento B150, puede estar relacionada con el efecto defoliante del exceso de este nutriente (Retamales, 2005).

\section{Contenido relativo de clorofilas}

Potasio. Se observaron diferencias significativas $(P \leq 0,05)$ entre el tratamiento K50 y los tratamientos K100 y K150 a los 135 ddt. A lo largo del ciclo pudo observarse que los valores de SPAD, para K50 fueron menores en relación con los de $\mathrm{K} 150$ y K100. El contenido relativo total de clorofila en las hojas de plantas con deficiencia de $\mathrm{K}$ fue 2,3\% menor al de las plantas control (Fig. 3A).

Los resultados de este estudio pese a no ser tan limitantes, pueden correlacionarse con lo encontrado en algodón (Gossypium hirsutum) por Zhao et al. (2001), quienes determinaron que ante deficiencia de $\mathrm{K}$ hubo una disminución del contenido de clorofilas en el 88\% respecto al control. Okanenko y Berstein (1969) por su parte, sugieren que el bajo contenido de clorofilas es debido a la pobre ultra estructuración del cloroplasto causada por la deficiencia de K. En contraste, Knypl y Rennert (1970) establecieron que el K, favorece la síntesis de clorofila a partir de mecanismos específicos, lo cual puede relacionarse con los mayores valores presentados por K100 y K150. 


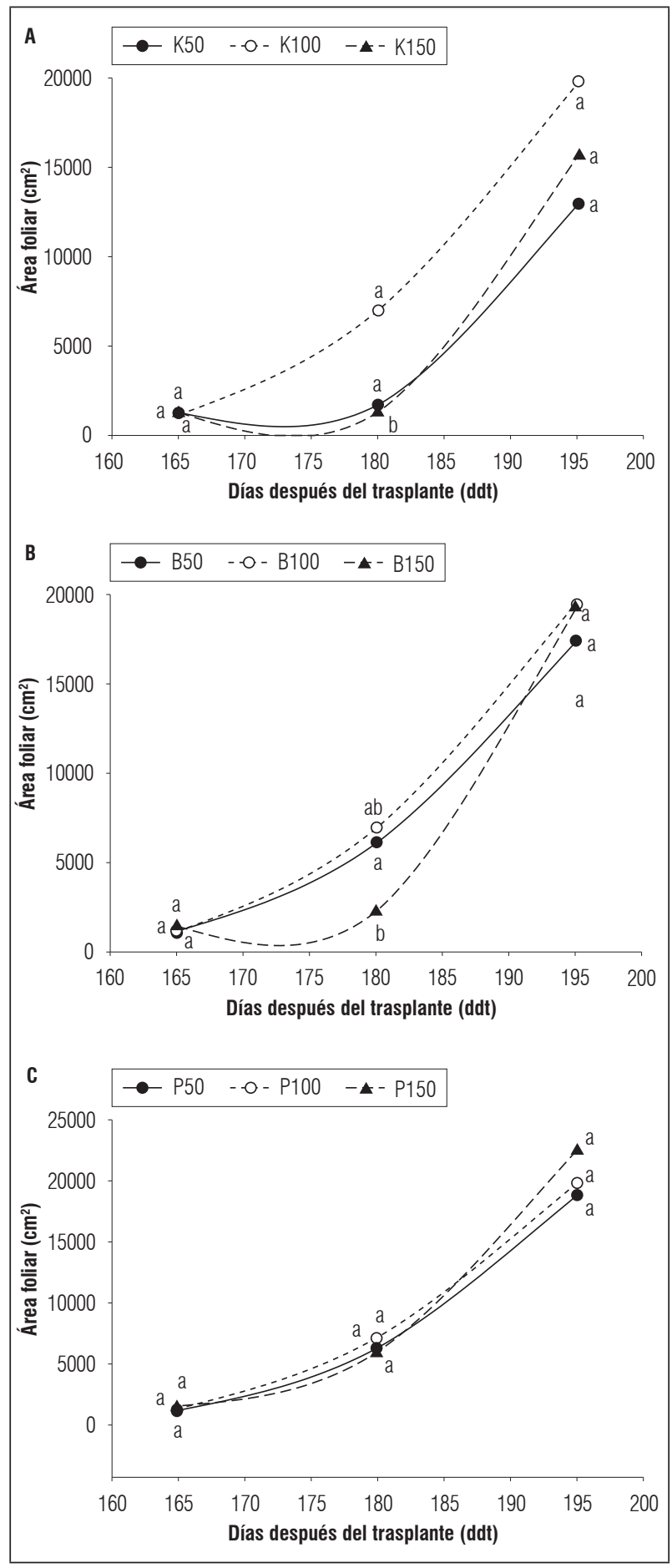

Figura 2. Deficiencias y excesos nutricionales sobre el área foliar de aguacate 'Hass'. A. Potasio (K); B. Boro (B) y C. Fósforo (P). Promedios con letras distintas indican diferencia significativa entre tratamientos, de acuerdo con la prueba de Duncan $(P \leq 0,05)$.
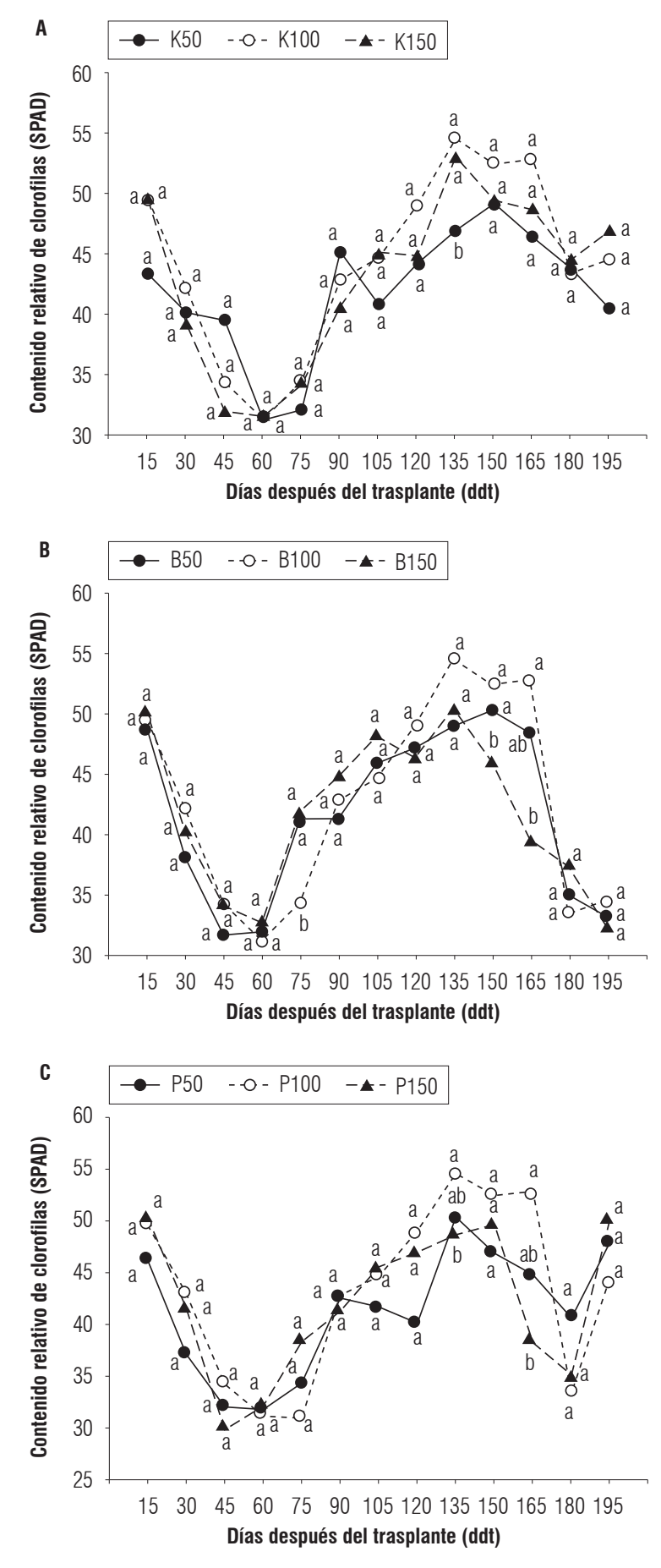

Figura 3. Deficiencias y excesos nutricionales sobre el contenido relativo de clorofilas (SPAD) de aguacate 'Hass'. A. Potasio (K); B. Boro (B) y C. Fósforo (P). Promedios con letras distintas indican diferencia significativa entre tratamientos, de acuerdo con la prueba de Duncan $(P \leq 0,05)$. 
Boro. Se observaron diferencias significativas $(P \leq 0,05)$ entre B100 y los tratamientos B50 y B150 a los $75 \mathrm{ddt}$ y entre B150 y B100 a los 150 y $165 \mathrm{ddt}$ (Fig. 3B).

Mouhtaridou et al. (2004) sugieren a partir de su estudio con manzana (Malus domestica), que el contenido de clorofilas decrece a medida que la concentración de $\mathrm{B}$ aumenta, lo cual puede asociarse con los menores valores presentados por B150, respecto a B100.

En forma complementaria, a lo largo del ciclo de evaluación se pudo observar que el tratamiento B50 (42,1 SPAD) presentó en promedio un contenido relativo de clorofilas de 3,5\% menor, en relación al de B100 (43,6 SPAD). Este resultado estaría en concordancia con Sharma y Ramchandra (1990), quienes encontraron que la deficiencia de $\mathrm{B}$ reduce el contenido de clorofilas y proteínas solubles en las hojas, lo que resulta en una inhibición de la reacción de Hill y la tasa de fotosíntesis neta.

Fósforo. Se observaron diferencias significativas $(P \leq 0,05)$ entre el tratamiento $\mathrm{P} 150$ y P100 a los 135 y 165 ddt. De acuerdo con Quintana et al. (2017) ante exceso de fósforo los contenidos de clorofila tienden a reducirse drásticamente debido a la ocurrencia de un antagonismo con aniones como el $\mathrm{NO}_{3}^{-}$ya que la capacidad fotosintética y, por ende, la clorofila, están relacionadas directamente con el contenido de fósforo y de nitrógeno en la planta (Singh y Reddy, 2015).

En adición, al analizar los resultados como consolidado, fue posible evidenciar que los valores para P50 (41,2 SPAD) fueron menores en un 5,5 \% al compararlos con los valores de P100 (43,6 SPAD) (Fig. 3C). Esta reducción de los pigmentos fotosintéticos, bajo la deficiencia fue consistente con otros estudios (Singh et al., 2013). Singh et al. (2017) sugieren que bajo deficiencia de P la disminución en el contenido de clorofilas se produce como un mecanismo para evitar el exceso de captación de luz, en pro de proteger el PSII del daño lumínico.

\section{Fluorescencia de la clorofila}

En la figura 4 no se observó un patrón claro de la Fv/ Fm para los diferentes tratamientos, que durante los primeros $90 \mathrm{ddt}$ los valores se alejaron ligeramente del valor de referencia 0,83 para plantas sin estrés (Percival, 2004; Kitajima y Butler, 1975). Esto podría indicar un ajuste de respuesta de la planta frente a las nuevas condiciones del sitio de evaluación. Un patrón similar se observó en el parámetro de transpiración (Fig. 5).

Potasio. Solamente se presentaron diferencias significativas $(P \leq 0,05)$ a los $195 \mathrm{ddt}$ entre K50 y los tratamientos K100 y K150 (Fig. 4A).

Aunque los efectos de la deficiencia de K son evidentes en variables relacionadas con el intercambio gaseoso, su relación con la eficiencia del PSII se conoce poco. Se reporta que la deficiencia de este elemento no tiene un efecto significativo sobre la eficiencia máxima potencial de las plantas (Saeed et al., 2009). De acuerdo con Salazar-García (2002), el exceso de potasio puede causar deficiencia de $\mathrm{Mg}$ y Yuan et al. (2016) describieron en plantas de calabaza (Cucurbita pepo) deficientes de $\mathrm{Mg}$ una disminución en Fv/Fm, lo cual atribuyeron a que el Mg está involucrado en la modulación de la actividad de enzimas fotosintéticas como la Rubisco (Laing et al., 2000). De igual forma, Ling et al. (2009), mencionan que las limitaciones no estomáticas pueden ser los principales factores que determinan la respuesta de la fotosíntesis cuando se presenta deficiencia de $\mathrm{Mg}$, ya que, la baja asimilación de $\mathrm{CO}_{2}$ está acompañada por un incremento de la concentración de $\mathrm{CO}_{2}$ intercelular. La deficiencia de $\mathrm{Mg}$ induce un deterioro en el transporte linear de electrones fotosintéticos, causando foto inhibición en las hojas por aumento de las especies reactivas de oxígeno, lo que conduce en un incremento de la emisión de luz en forma de fluorescencia para proteger el PSII.

Boro. Únicamente se presentaron diferencias significativas $(P \leq 0,05)$ a los 15 ddt entre B50 y B150 (Fig. 4B). Momento para el cual B50 presentó valores 2,1 y 7,9\% menores a los exhibidos por B100 y B150, respectivamente. Estos resultados son acordes con lo descrito por Dell y Huang (1997) quienes indican que la deficiencia de B puede afectar indirectamente la fotosíntesis porque reduce el área fotosintéticamente activa y altera los constituyentes foliares.

Pese a que los resultados del experimento no demostraron de forma clara el efecto del exceso de B sobre la relación Fv/Fm, autores como Gimeno et al. (2012) reportan que los mayores niveles de $\mathrm{B}$ generan una reducción significativa de la relación Fv/Fm. Ellos atribuyen este comportamiento a la pérdida de la eficiencia cuántica del PSII, debido a un daño en el complejo captador de luz (Guerfel et al., 2009). Asimismo, Papadakis et al. (2004) explican que otra de las consecuencias del exceso de B en las hojas, es el daño en la estructura del cloroplasto, lo cual tiene un efecto directo sobre la tasa de fotosíntesis. 
Fósforo. Se observaron diferencias significativas $(P \leq 0,05)$ entre $\mathrm{P} 150$ y $\mathrm{P} 100$ a los $45 \mathrm{ddt}$. Momento en el cual P150 mostró valores para Fv/Fm 6,3\% mayores a los presentados por P100 (Fig. 4C).

Es sabido que el $\mathrm{P}$ no afecta directamente la fotosíntesis, pero es un nutriente fundamental en el metabolismo de las plantas, como componente de ácidos nucleicos y de moléculas ricas en energía (Jiménez et al., 2015). A pesar de ello, según Salazar-García (2002), el exceso de fósforo puede ocasionar deficiencias de manganeso (Mn). Roosta et al. (2017) encontraron en plantas de lechuga (Lactuca sativa), que la relación Fv/ Fm fue significativamente afectada por la deficiencia de este microelemento. Este efecto ocurre como resultado de los daños a los centros de reacción del PSII, por el incremento de las cantidades de electrones y radicales libres. De igual forma, Qu et al. (2012) afirman que el estrés por deficiencia de $\mathrm{Mn}$ disturba severamente el transporte de electrones o genera un daño en la estructura de la membrana tilacoidal en el lado donador del PSII.

Por otra parte, y a pesar de la ausencia de diferencias significativas en el comportamiento de P50 respecto al de P100, es importante agregar que la deficiencia de $\mathrm{P}$, puede llegar a generar una disminución en Fv/Fm, por la producción de una fotoinhibición en las hojas (Singh et al., 2017). La fotoinhibición usualmente resulta en una reducción de la eficiencia cuántica relativa del PSII, y ésta en una reducción en el transporte de electrones, lo cual puede atribuirse a una disminución del número de centros de reacción del PSII y la eficiencia en la captura de energía por estos centros (He et al., 2011).

\section{Transpiración}

Potasio. Se presentaron diferencias significativas $(P \leq 0,05)$ entre K100 y K50 a los 30 ddt y entre K150 y los tratamientos K100 y K50 a los 150 ddt (Fig. 5A). Haciendo referencia al efecto de la deficiencia de K, Behboudian y Anderson (1990) en plantas de tomate (Solanum lycopersicum), y Zhao et al. (2001), en plantas de algodón (Gossypium hirsutum), determinaron, que esta genera una disminución significativa de la tasa de transpiración, lo cual se traduce en una supresión del crecimiento. Adicionalmente, Tang et al. (2015) indicaron que existe evidencia sustancial, para afirmar que el daño en la estructura de los cloroplastos causada por la deficiencia de $\mathrm{K}$, altera la regulación estomatal y no estomatal de $\mathrm{CO}_{2}$ y el flujo de $\mathrm{H}_{2} \mathrm{O}$ en

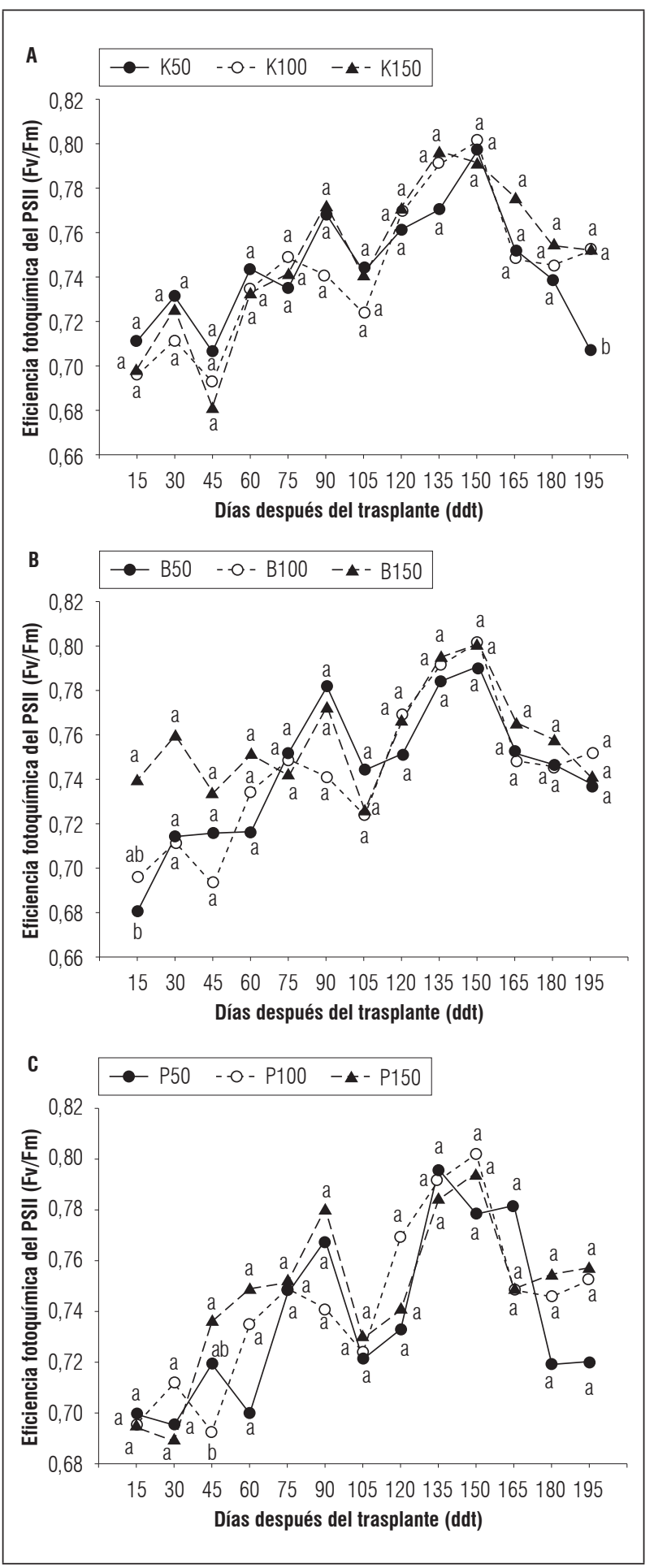

Figura 4. Deficiencias y excesos nutricionales sobre la eficiencia máxima fotoquímica del PSII (Fv/Fm) en aguacate 'Hass'. A. Potasio (K); B. Boro (B) y C. Fósforo (P). Promedios con letras distintas indican diferencia significativa entre tratamientos, de acuerdo con la prueba de Duncan $(P \leq 0,05)$. 
las hojas, limitando así la tasa transpiratoria. Sin embargo, para el caso específico de este estudio, se pudo establecer que la deficiencia de $\mathrm{K}$ al $50 \%$, no generó un detrimento considerable de la tasa de traspiración, debido probablemente a que los niveles de $\mathrm{K}$ no fueron lo suficientemente bajos como para promover esta reacción.

De igual forma, al observar el comportamiento de las plantas ante el exceso de $\mathrm{K}$ a los $150 \mathrm{ddt}$, se evidencia que estas presentaron valores significativamente mayores en relación con los del control. Este resultado puede correlacionarse con lo encontrado por Farhat et al. (2013) en plantas de girasol (Helianthus annuus), donde se establece que tanto por efecto del exceso K como por el efecto de deficiencia de $\mathrm{Mg}$, pueden ocurrir alteraciones en la tasa de transpiración.

Boro. Se observaron diferencias significativas $(P \leq 0,05)$ entre B100 y los tratamientos B150 y B50 a los 30 ddt. Y entre B50 y los tratamientos B100 y B150 a los 150 ddt. (Fig. 5B). No obstante, la mayor transpiración presentada por B50 a los 150 ddt, está en contra posición con lo descrito por Mukhopadhyay et al. (2013) en plantas de té (Camellia sinensis), sometidas a deficiencia de B. Puesto que, ellos establecieron que, ante deficiencia de $\mathrm{B}$ se produce un decrecimiento de la tasa de transpiración, la cual se relaciona con una baja conductancia estomática, causada por el alto daño oxidativo en las hojas. Por lo cual, concluyen que todos los parámetros relacionados con el intercambio gaseoso son influenciados por la deficiencia del nutriente. Adicionalmente, sugieren que la aparición de hexosas libres puede desencadenar una baja regulación del ciclo de Calvin y por ende inhibir las tasas de fotosíntesis.

En contraste, cuando se analizan los resultados presentados por B150 se observa que están en concordancia con lo expuesto por Shani et al. (1992), quienes reportan que el exceso de $B$ no genera una reducción significativa en la transpiración. Pero que, si la concentración de $\mathrm{B}$ aumenta exponencialmente, la tasa transpiratoria comenzará a verse afectada (Ben-Gal y Shani, 2003).

Fósforo. Se evidenciaron diferencias significativas $(P \leq 0,05)$ entre P150 y los tratamientos P100 y P50 a los 15 ddt, y entre P50 y P100 a los 60 dtt (Fig. 5C). La menor tasa de transpiración expuesta por P150 a los $15 \mathrm{ddt}$, puede asociarse al efecto del bloqueo de la asimilación de $\mathrm{K}$, causado por un aumento del flujo de Mg ante exceso de P (Navarro y Navarro, 2014).

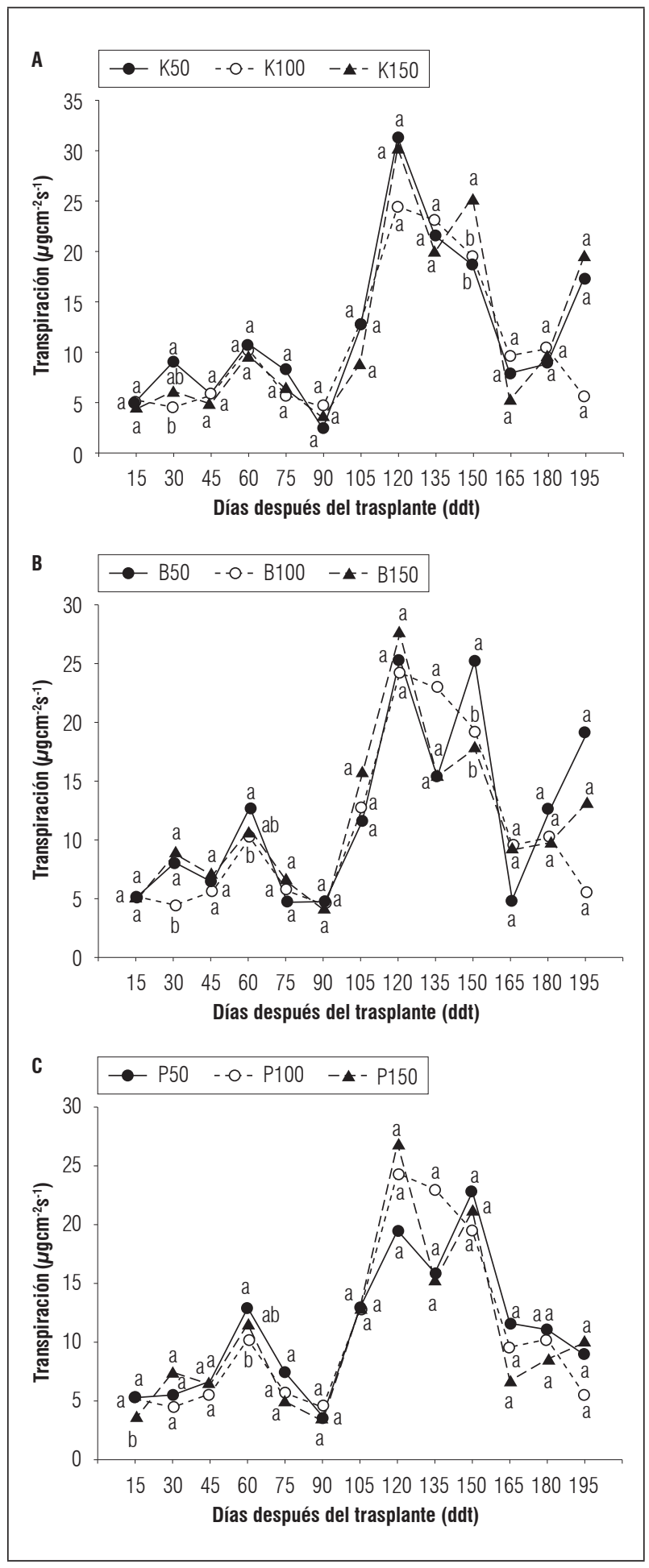

Figura 5. Deficiencias y excesos nutricionales sobre la transpiración de aguacate 'Hass'. A. Potasio (K); B. Boro (B) y C. Fósforo (P). Promedios con letras distintas indican diferencia significativa entre tratamientos, de acuerdo con la prueba de Duncan $(P \leq 0,05)$. 
Lo cual se explica a través del papel que ejerce el $\mathrm{K}$ sobre la regulación estomatal y no estomatal de $\mathrm{CO}_{2}$ y el flujo de $\mathrm{H}_{2} \mathrm{O}$ en las hojas (Tang et al., 2015).

A su vez, también es posible establecer que el comportamiento de P50 frente a P100, discierne de lo reportado en la literatura. Veronica et al. (2017) afirman que la deficiencia de $\mathrm{P}$ resulta en una reducción de la tasa de transpiración, así como de la conductancia estomática y la concentración intercelular de $\mathrm{CO}_{2}$, lo cual atribuyeron a una limitación estomática.

\section{Sintomatología}

Potasio. Los síntomas de la deficiencia se presentaron únicamente en hojas bajeras, primero en forma de una banda amarillenta ancha cerca del borde, seguida de otra verde claro que dejó ver áreas pequeñas más oscuras de bordes indefinidos (Fig. 6A). Posteriormente estas mostraron las venas amarillas y áreas necróticas café rojizo desde los bordes hacia el interior de la lámina. En algunos casos se presentó necrosis en los bordes de las hojas limitada por un halo verde amarillento. Las hojas jóvenes presentaron clorosis leve (Fig. 6B y 6C).

De acuerdo con Salazar-García (2002), el exceso de potasio puede causar deficiencias de Ca o Mg. En las plantas sometidas a exceso fue posible observar una clorosis leve, que inició en el ápice o por la zona media de la hoja y avanzó por los bordes hacia toda la lámina (Fig. 7A y 7B). Lo cual puede asociarse a síntomas relacionados con deficiencia $\mathrm{Mg}$, similares a lo descrito por Navarro y Navarro (2000). En algunas plantas se observaron síntomas asociados a clorosis de la lámina foliar, acompañados de puntos necróticos que coalecen en los bordes sin un patrón evidente (Fig. 7C y D).

Boro. En general, las plantas deficientes en boro presentaron los primeros síntomas en las hojas jóvenes; estas se tornaron más pequeñas, flácidas y perdieron el brillo. Cuando la deficiencia avanzó, se presentaron deformaciones en la lámina. Adicionalmente, se presentó clorosis que inició en el ápice de las hojas. Por el contrario, en hojas adultas se observó una coloración verde claro ligeramente mate (Fig. 8).

Ante el exceso, pudo observarse necrosis apicales y un aumento de tamaño en la lámina foliar (Fig. 9), esto quizás asociado a un aumento en el nivel de RNA y, en consecuencia, un incremento de la división celular en los meristemos (Wild y Jones, 1992), esto por el papel del elemento en la elongación celular y la síntesis de ácidos nucleicos (Taiz y Zeiger, 2010).

Fósforo. Los síntomas de deficiencia se manifestaron como una clorosis difusa de tono opaco en las hojas adultas (Fig. 10A), esta evolucionó como manchas necróticas sin patrón definido (Fig. 10C-E). La textura de las hojas se tornó coriácea, mientras que el color se mantuvo verde muy oscuro sin brillo. La venación se conservó de color verde oscuro. No se observó clorosis generalizada en hojas jóvenes ni en el resto de la planta. Sin embargo, se presentó una leve evidencia de acumulación de antocianinas (Fig. 10B).

De acuerdo con Salazar-García (2002) el exceso de fósforo puede manifestarse como deficiencias de micronutrientes como Mn y/o Zn.
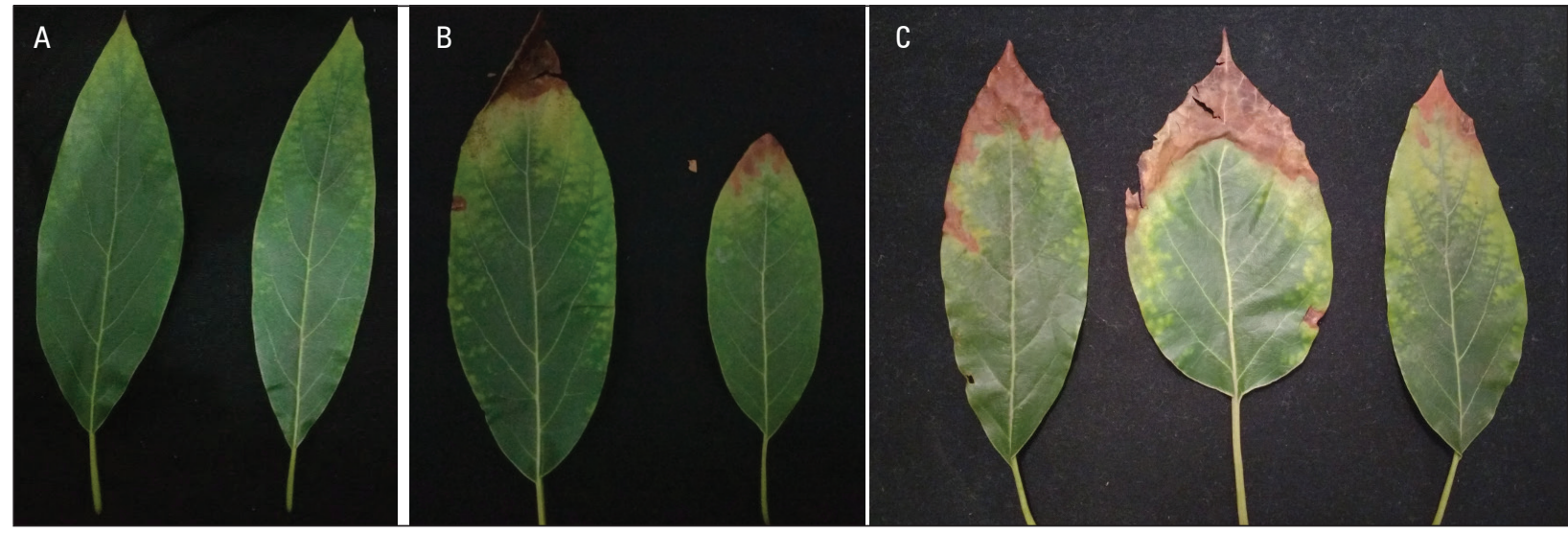

Figura 6. Síntomas de deficiencia de potasio en hojas de plantas de aguacate var. Hass. A. Clorosis en forma de banda; B y C. Avance de necrosis en hojas adultas. 

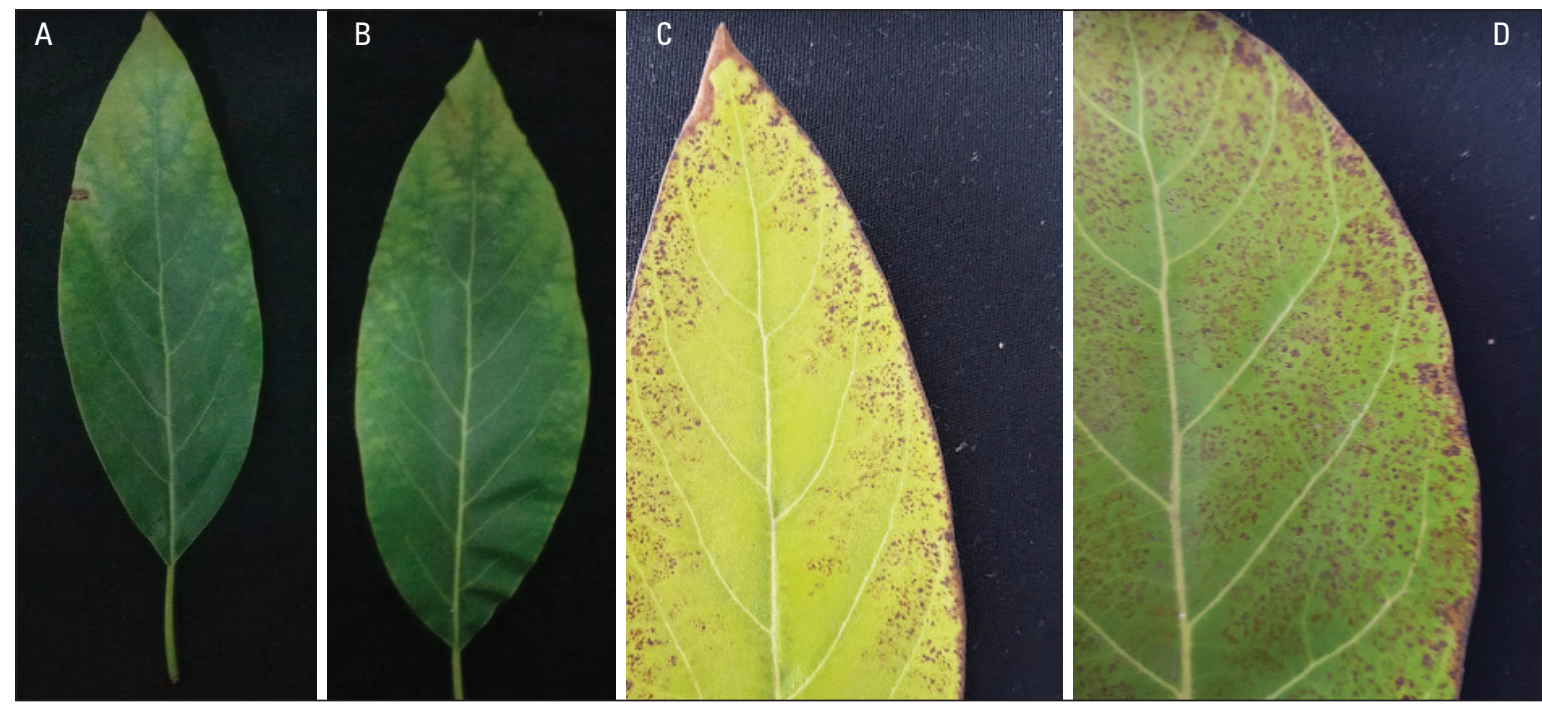

Figura 7. Síntomas de exceso de potasio en hojas de plantas de aguacate var. Hass. A y B. Clorosis en hojas adultas; C y D. Puntos necróticos sin patrón evidente.
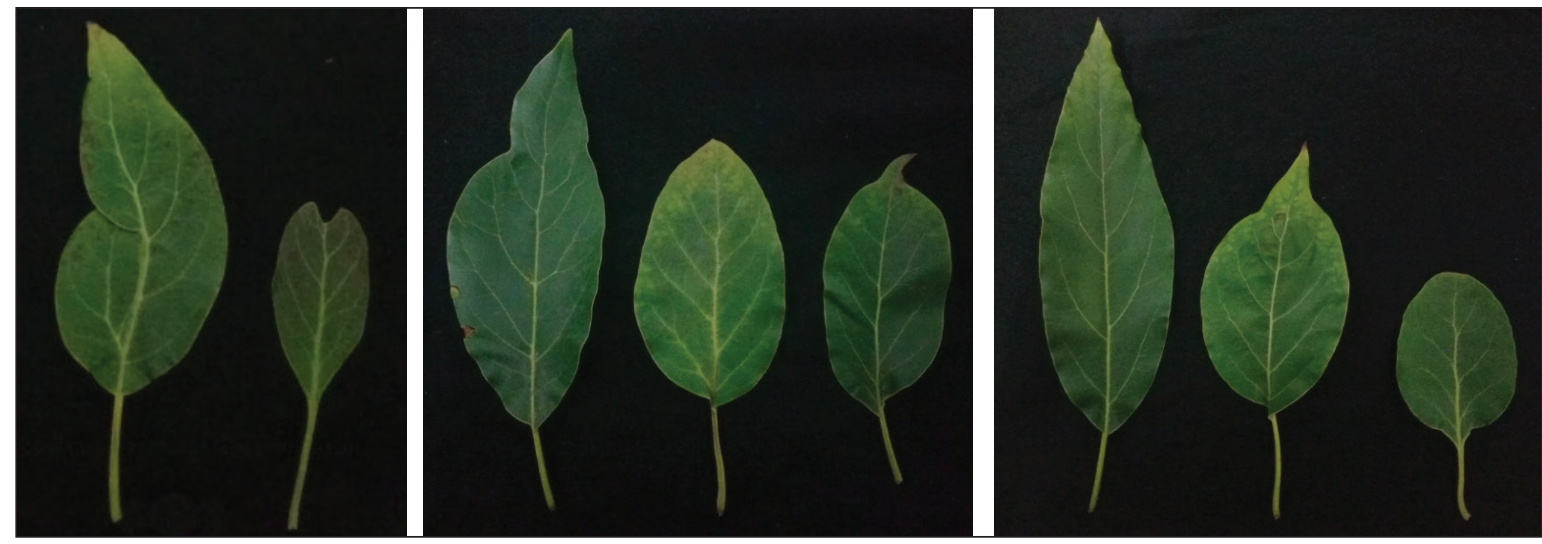

Figura 8. Síntomas de deficiencia de boro en hojas de plantas de aguacate var. Hass.
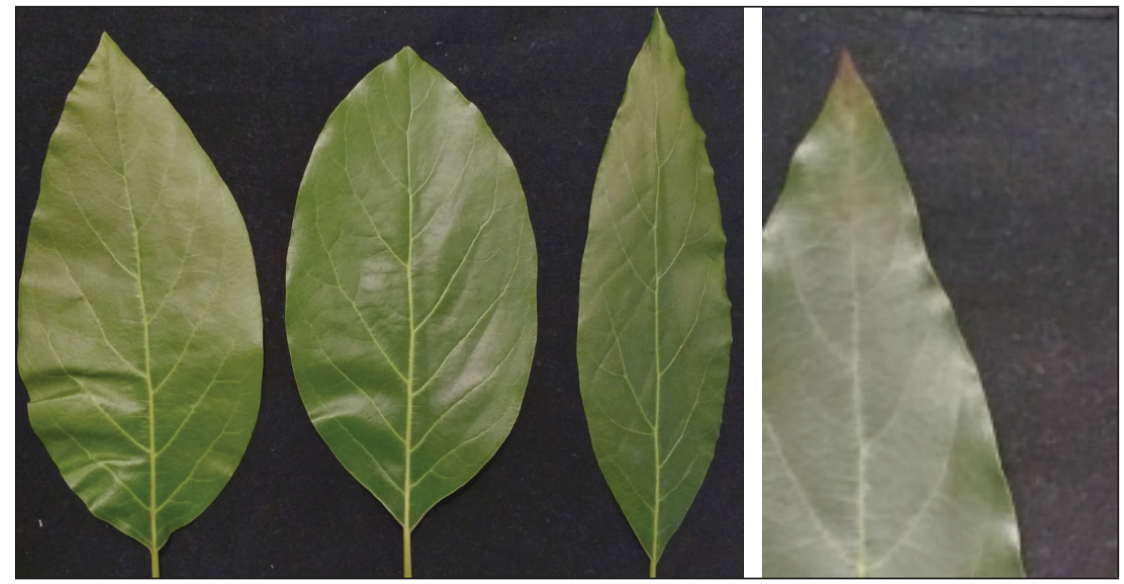

Figura 9. Síntomas de exceso de boro en hojas de plantas de aguacate var. Hass. 



Figura 10. Síntomas de deficiencia de fósforo en hojas de plantas de aguacate var. Hass. A. Clorosis en hojas adultas; B. Acumulación de antocianinas en hojas jóvenes; C., D. y E. Manchas necróticas sin patrón definido.

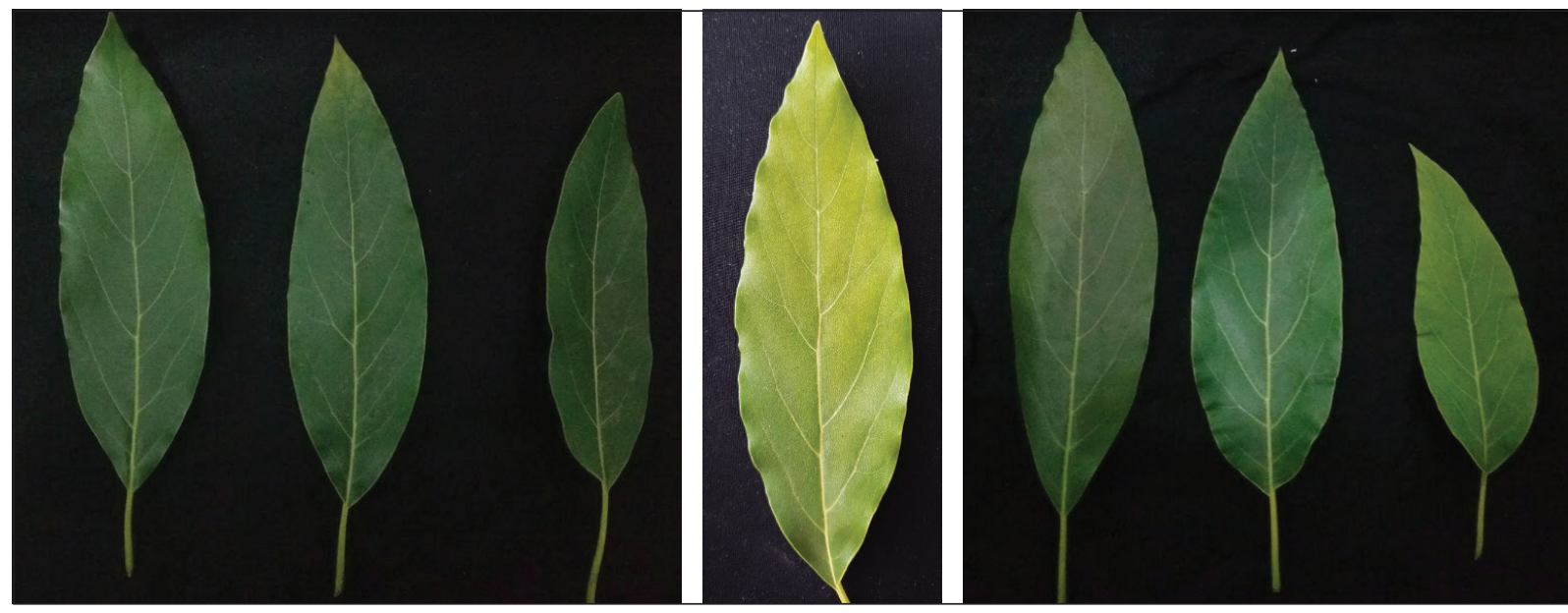

Figura 11. Síntomas de exceso de fósforo en hojas de plantas de aguacate var. Hass. Hojas jóvenes cloróticas y con crecimiento lanceolado. 
En las plantas sometidas a exceso de $\mathrm{P}$ pudo observarse síntomas de clorosis en hojas jóvenes, no muy intensa y extendida en la lámina de la hoja. Lo cual coincidiría con lo descrito por Sierra (2003), para los síntomas de deficiencia de Mn. Adicionalmente pudo evidenciarse un crecimiento algo lanceolado y reducido en hojas nuevas (Fig. 11). Hernández y Pacheco (1986) describen estos síntomas para la deficiencia de Zn en cardamomo (Elettaria cardamomum).

\section{CONCLUSIONES}

Las plantas de aguacate var. Hass en fase vegetativa del crecimiento, bajo condiciones de invernadero de plástico y sometidas a deficiencia de potasio o exceso de boro, presentaron los menores valores para las variables número de hojas y área foliar en comparación con el control.

En cuanto a parámetros relacionados con fotosíntesis e intercambio de gases, se observó que los tratamientos de deficiencia y exceso de los nutrientes evaluados no presentaron diferencias significativas consistentes a través del tiempo, respecto al control. Esto podría estar sugiriendo que la deficiencia al $50 \%$ y el exceso al $150 \%$, no son lo suficientemente limitantes como para generar un detrimento considerable de dichos procesos fisiológicos. Adicionalmente, estos resultados constituirían una evidencia para considerar que la dosis comercial no provee los niveles óptimos de nutrientes, puesto que las plantas a lo largo del ciclo no expusieron los valores de referencia.

Finalmente, al referirse a la expresión de síntomas visuales de estrés nutricional, es posible indicar que esta ocurrió de forma más evidente en plantas sometidas a deficiencias, que en las sometidas a excesos. Esto último posiblemente asociado a que, al no ser la dosis comercial aquella que proveyera los niveles óptimos de nutrientes, la dosis al 150\% no estaría representado realmente un exceso.

\section{AGRADECIMIENTOS}

A Colciencias por la financiación de este trabajo de grado (M.A. Novoa-Torres) enmarcado en el proyecto "Ecofisiología, nutrición mineral y manejo integrado de plagas y enfermedades en aguacate, curuba, gulupa y tomate de árbol orientados hacia su manejo agronómico, como materia prima para el desarrollo de productos de interés comercial" coordinado por L.M.
Melgarejo, de la Red Nacional para la bioprospección RIFRUTBIO contrato 459/2013. Al Departamento de Biología y la Facultad de Ciencias Agrarias de la Universidad Nacional de Colombia, sede Bogotá.

Conflicto de intereses: el manuscrito fue preparado y revisado con la participación de los autores, quienes declaran no tener algún conflicto de interés que coloquen en riesgo la validez de los resultados aquí presentados.

\section{REFERENCIAS BIBLIOGRÁFICAS}

Agronet. 2016. Aguacate Hass colombiano entra en la recta final para exportar a Estados Unidos. En: Agronet, https://www.minagricultura.gov.co/noticias/Paginas/Aguacate-Hass-colombiano-entra-en-la-recta-final-para-exportar-a-Estados-Unidos.aspx; consultado: octubre de 2017.

Bartoli, J. 2008. Manual técnico del cultivo de aguacate Hass (Persea americana L.). Centro de Comunicación Agrícola de la Fundación Hondureña de Investigación Agrícola (FHIA), Lima, Perú.

Behboudian, M. y D. Anderson. 1990. Effects of potassium deficiency on water relations and photosynthesis of the tomato plant. Plant Soil 127, 137-139. Doi: 10.1007/BF00010846

Ben-Gal, A. y U. Shani. 2003. Water use and yield of tomatoes under limited water and excess boron. Plant Soil 256, 179-186. Doi: 10.1023/A:1026229612263

Bernal, J. y C. Díaz. 2005. Tecnología para el cultivo del Aguacate. Manual Técnico No. 5. Colombia: Centro de Investigación La Selva, Corporación Colombiana de Investigación Agropecuaria (Corpoica), Rionegro, Antioquia.

Dell, B. y L. Huang. 1997. Physiological response of plants to low boron. Plant Soil 193, 103-120. Doi: 10.1023/A:1004264009230

Fairhurst, T. 1999. Nutrient use efficieny in oil palm: measurement and management. The Planter 75, 173-177.

FAO. 2016. FAOSTAT statistics database. En: http://www. fao.org/statistics/es/; consultado: octubre de 2017.

Farhat, N., M. Rabhi, H. Falleh, K. Lengliz, A. Smaoui, C. Abdelly y M. Lachaal. 2013. Interactive effects of excessive potassium and $\mathrm{Mg}$ deficiency on safflower. Acta Physiol. Plant 35, 2727-2745. Doi: 10.1007/ s11738-013-1306-x

Gimeno, V., L. Simón, M. Nieves, V. Martínez y J. Camara. 2012. The physiological and nutritional responses to an excess of boron by Verna lemon trees that were 
grafted on four contrasting rootstocks. Trees 26, 15131526. Doi: 10.1007/s00468-012-0724-5

Guerfel, M., O. Baccouri, D. Boujnah, W. Chaibi y M. Zarrouk. 2009. Impacts of water stress on gas exchange, water relations, chlorophyll content and leaf structure in the two main Tunisian olive (Olea europea L.) cultivars. Sci. Hortic. 119, 257-263. Doi: 10.1016/j. scienta.2008.08.006

He, G., J. Zhang, X. Hu y J. Wu. 2011. Effect of aluminum toxicity and phosphorus deficiency on the growth and photosynthesis of oil tea (Camellia oleifera Abel.) seedlings in acidic red soils. Acta Physiol. Plant. 33, 12851292. Doi: 10.1007/s11738-010-0659-7

Hernández, R. y R. Pacheco. 1986. Caracterización de síntomas visuales de deficiencias nutricionales en cardamomo (Elettaria cardamomum). Agron. Costarr. 10(1/2), 13-27.

IPNI (International Plant Nutrition Institute). 2010. Funciones del fosforo en las plantas. Informaciones Agronomicas No. 36. IPNI, Peachtree Corners, GA, USA.

ICA (Instituto Colombiano Agropecuario). 2012. Manejo fitosanitario del cultivo del aguacate Hass (Persea americana Mill): medidas para la temporada invernal. Bogotá, Colombia.

Jiménez, S., O. Alvarado y H. Balaguera. 2015. Fluorescencia como indicador de estrés en Helianthus annuus L. Una revisión. Rev. Colomb. Cienc. Hortic. 9(1), 149160. Doi: $10.17584 /$ rcch.2015v9i1.3753

Jordan, L. y S. Pellerin. 2004. Leaf area establishment of a maize (Zea mays L.) field crop under potassium deficiency. Plant Soil 265, 75-92. Doi: 10.1007/ s11104-005-0695-z

Kass, D. 1998. Fertilidad de suelos. Ed. Universidad Estatal a Distancia. San José, Costa Rica.

Kitajima, M. y W. Butler. 1975. Quenching of chlorophyll fluorescence and primary photochemistry in chloroplasts by dibromothymoquinone. Biochim. Biophys. Acta 376, 105-115. Doi: 10.1016/0005-2728(75)90209-1

Knypl, J. y A. Rennert. 1970. Stimulation of growth and chlorophyll synthesis in detached cotyledons of $\mathrm{cu}$ cumber by potassium. Z. Pflanzenphysiol. 62, 97-107.

Laing, W., D. Greer, O. Sun, P. Beets, A. Lowe y T. Payn. 2000. Physiological impacts of $\mathrm{Mg}$ deficiency in Pinus radiata: growth and photosynthesis. New Phytol. 146, 47-59. Doi: 10.1046/j.1469-8137.2000.00616.x

Ling, L., L. Peng, L. Cao, C. Jiang, C. Chun, G. Zhang y Z. Wang. 2009. Effect of magnesium deficiency on photosynthesis characteristic of Beibei 447 Jinchen orange. J. Fruit Sci. 26, 275-280.

Maldonado, R., M. Álvarez, G. Almaguer, A. Barrientos y R. García. 2013. Estándares nutrimentales para aguacatero "Hass". Rev. Chapingo Ser. Hortic. 13(1), 103-108.
Mejía, A. 2010. Cadena Productiva del Aguacate en Colombia. Consejo Nacional del Aguacate. pp. 5-30. En: Memorias, II Encuentro de la Cadena Productiva del Aguacate. Corporción Antioqueña del Aguacate, Ríonegro, Colombia.

Mouhtaridou, G., T. Sotiropoulos, K. Dimassi y I. Therios. 2004. Effects of boron on growth, and chlorophyll and mineral contents of shoots. Biol. Plant. 48(4), 617-619. Doi: 10.1023/B:BIOP.0000047169.13304.67

Mukhopadhyay, M., P. Ghosh y T. Mondal. 2013. Effect of boron deficiency on photosynthesis and antioxidant responses of young tea plantlets. Russ. J. Plant Physiol. 60(5), 633-639. Doi: 10.1134/S1021443713030096

Navarro, S. y G. Navarro. 2000. Química agrícola. Ed. Mundi-Prensa, Madrid, España.

Navarro, S. y G. Navarro. 2014. Fertilizantes: química y acción. Ed. Mundi-Prensa, Madrid, España.

Okanenko, A. y B. Berstein. 1969. Potassium, photosynthesis and phosphate metabolism in sugar-beet. Naukova Dumka, Kiev, Ucracia.

Olivia, M., N. de Barros y M. de Mouza. 1995. Muerte apical en eucalipto y manejo nutritivo de plantaciones forestales: aspectos fisiológicos del problema. Bosque 16(1), 77-86. Doi: 10.4206/bosque.1995.v16n1-08

Papadakis, I., N. Dimassi, A. Bosabalidis, L. Therios, A. Patakas y A. Giannakoula. 2004. Boron toxicity in Clementine mandarin plants on two rootstocks. Plant Sci. 166, 539-547. Doi: 10.1016/j.plantsci.2003.10.027

Percival, G. 2004. Evaluation of physiological tests as predictors of young tree establishment and growth. J. Arboric. 30(2), 80-92.

Pillimue, G., N. Barrera y S. de Cantillo. 1998. Determinación de deficiencias de elementos mayores en plantas de tomate de árbol. Acta Agron. 48(3/4), 62-67.

Quintana, W., E. Pinzón y D. Torres. 2017. Efecto de un fosfato térmico sobre el crecimiento y producción de fríjol (Phaseolus vulgaris L.) cv. ICA Cerinza. Rev. UDCA Act. \& Div. Cient. 20(1), 51-59.

Qu, C., X. Gong, C. Liu, M. Hong, L. Wang y F. Hong. 2012. Effects of manganese deficiency and added cerium on photochemical efficiency of maize chloroplasts. Biol. Trace Elem. Res. 146, 94-100. Doi: 10.1007/ s12011-011-9218-3

Retamales, J. 2005. Fisiología y manejo de la nutrición de boro, potasio y calcio en pomáceas. Rev. Unifrut 15, 10-20.

Ríos, D. y R. Tafur. 2003. Variedades de aguacate para el trópico: caso Colombia. pp. 143-147. En: Actas, V Congreso Mundial del Aguacate. Granada, Málaga, España.

Roosta, H., A. Estaji y F. Niknam. 2017. Effect of iron, zinc and manganese shortage-induced change on photosynthetic pigments, some osmoregulators and 
chlorophyll fluorescence parameters in lettuce. Photosynthetica 55(10), 1-10.

Saeed, M., M. Ashraf, M. Shahbazy A. Aisha. 2009. Growth and photosynthesis of salt-stressed sunflower (Helianthus annuus) foliar applied different potassium salts. J. Plant Nutr. Soil Sci. 172(6), 884-893. Doi: 10.1002/ jpln.200900102

Salazar, S., L. Cossio y I. González. 2009. La fertilización de sitio específico mejoró la productividad del aguacate 'Hass' en huertos sin riego. Agric. Téc. Mex. 35(4), 439-448.

Salazar-García, S. 2002. Nutrición del aguacate, principios y aplicaciones. Instituto Nacional de Investigaciones Forestales, Agrícolas y Pecuarias (INIFAP); Instituto de la Potasa y el Fósforo (INPOFOS), Querétaro, México.

Salazar, S. y I. Lazcano. 2003. Site-specific fertilization increased yield and fruit size in 'hass' avocado. Better Crops Int. 17(1), 13-15.

Salas, R. 2002. Herramientas de diagnóstico para definir recomendaciones de fertilización foliar. Centro de Investigaciones Agronómicas, San José, Costa Rica.

Shani, U., L. Dudley y R. Hanks. 1992. Model of boron movement in soils. Soil Sci. Soc Am. J. 56(5),1365-1370. Doi: 10.2136/sssaj1992.03615995005600050006x

Sharma, P. y T. Ramchandra. 1990. Water relations and photosynthesis in mustard plants subjected to boron deficiency. Indian J. Plant Physiol. 33, 150-154.

Sierra, C. 2003. Fertilización de cultivos y frutales en la zona norte. Boletin INIA No. 97. Instituto de Investigaciones Agropecuarias, La Serena, Chile.

Sindoni, M., J. Zamora y R. Ramírez. 1994. Síntomas de deficiencia de boro y producción de materia seca en ajonjolí. Agron. Trop. 44(1), 135-150.

Singh, S., G. Badgujar y V. Reddy. 2013. Carbon dioxide diffusion across stomata and mesophyll and photo-biochemical processes as affected by growth $\mathrm{CO}_{2}$ and phosphorus nutrition in cotton. J. Plant Physiol. 170, 801-813. Doi: 10.1016/j.jplph.2013.01.001

Singh, S. y V. Reddy. 2015. Response of carbon assimilation and chlorophyll fluorescence to soybean leaf phosphorus across $\mathrm{CO}_{2}$ : Alternative electron sink, nutrient efficiency and critical concentration. J. Photochem. Photobiol. B: Biology 151, 276-284. Doi: 10.1016/j.jphotobiol.2015.08.021

Singh. S., V. Reddy, D. Fleishery D. Timlin. 2017. Relationship between photosynthetic pigments and chlorophy11 fluorescence in soybean under varying phosphorus nutrition at ambient and elevated $\mathrm{CO}_{2}$. Photosynthetica 55(3), 421-433. Doi: 10.1007/s11099-016-0657-0

Taiz, L. y E. Zeiger. 2010. Plant physiology. 5a ed. Sinauer Associates, Sunderland, MA, USA.

Tang, Z., J. Zhang, M. Wei, X. Chen, Z. Liu, H. Li e Y. Ding. 2015. Physiological response to potassium deficiency in three sweet potato (Ipomoea batatas [L.] Lam.) genotypes differing in potassium utilization efficiency. Acta Physiol. Plant. 37(184), 1-10. Doi: 10.1007/ s11738-015-1901-0

Veronica, N., D. Subrahamanyam, T. Vishnu, P. Yugandhar, V. Bhadana, V. Padma, G. Jayasree y S. Voleti. 2017. Influence of low phosphorus concentration on leaf photosynthetic characteristics and antioxidant response of rice genotypes. Photosynthetica 55(2), 285-293. Doi: 10.1007/s11099-016-0640-4

Wild, A. y J. Jones. 1992. Nutrición mineral de las plantas cultivadas. pp. 73-119. En: Wild, A. (ed.). Condiciones del suelo y desarrollo de las plantas según Russel. Ed. Mundi-Prensa, Madrid, España.

Yabrudy, J. 2012. El aguacate en Colombia: Estudio de caso de los Montes de María, en el Caribe colombiano. Documentos de Trabajo sobre Economía Regional y Urbana 171. Banco de la República, Bogotá, Colombia.

Yuan, H., J. Yanyan, A. Muhammad, C. Chen, L. Li, L. Zhen, K. Qiusheng, C. Fei y B. Zhilong. 2016. Improving magnesium uptake, photosynthesis and antioxidant enzyme activities of watermelon by grafting onto pumpkin rootstock under low magnesium. Plant Soil 409, 229-246. Doi: 10.1007/s11104-016-2965-3

Zhao, D., D. Oosterhuis y C. Bednarz. 2001. Influence of potassium deficiency on photosyntesis, chorophylll content, and chloroplast ultrastructure of cotton plants. Photosynthetica 39(1), 103-109. Doi: 10.1023/A:1012404204910 